\title{
Edge Detection Using Simplified Second Order Differential Hyperbolic Tangent Steerable Filter
}

\author{
${ }^{1} \mathrm{~S}$. Anand, ${ }^{1} \mathrm{R}$. Shantha Selva Kumari and ${ }^{2} \mathrm{H}$. Abirami \\ ${ }^{1}$ Department of Electronics and Communication Engineering, \\ Mepco Schlenk Engineering College, Sivakasi, Tamil Nadu, India \\ ${ }^{2}$ Department of Electronics and Communication Engineering, \\ VV College of Engineering, Tirunelveli, Tamil Nadu, India
}

\begin{abstract}
This study presents a new Edge Detection (ED) in images using simplified Second order Differential Hyperbolic Tangent (SDHT) steerable filter. Smoothing the image with Gaussian filter is the 1st step in conventional ED algorithm. However, shape or smooth parameter of the Gaussian filter influences the overall performance of ED. In particular when the smoothing parameter is $>1$, Gaussian filter provides excess smoothness and get widened and dislocated edges. This new SDHT filter provides better trade-off between a level of smoothing and edge feature detection than Gaussian filter. This new algorithm not only captures multiple orientations but also well in ED, improved noise tolerance with less computational cost. Mean Squared Error (MSE) and Peak Signal to Noise Ratio (PSNR) parameters are used to measure the quantitative performance of detection and noise tolerance. Experimental results show that the average MSE of 44 different images is reduced in proposed algorithm (0.299975) while compared with Canny (0.30408) and Sobel (0.33152). While filtering the edge information, PSNR is improved in proposed SDHT (57.5) than Gaussian (56.0).
\end{abstract}

$\underline{\text { Key words: Edge detection, hyperbolic tangent filter, steerable filter, algorithm, parameters, India }}$

\section{INTRODUCTION}

Edge is a distinct observable variation in the intensity function and image intensity is often proportional to physical edges that correspond to the significant variations in reflectance, illumination, orientation and depth of scene surfaces. For many images, the essential information processing closely linked with important edge features. Due to information wealth associated with edges, Edge Detection (ED) algorithms have important task in many applications related to pattern recognition and computer vision. Since an image is a discrete data, the edges are often defined as the local maxima of the derivative(s). The classical 2-D edge detection algorithms based on gradient-based operators such as Roberts, Prewitt, Sobel and Fri-Chen are high pass filter in nature and sensitive to noise. To combat with noise, more general robust extension algorithms are devised (Marr and Hildreth, 1980). This approach combines Gaussian smoothing while estimating the gradient information. They are more effective in higher noise conditions at the cost of dislocation and suppression of weak edge features and are attractive due to low complexity linear implementation. In order to achieve simultaneous detection and localization of edge pixels, there is a need for tradeoff between the smoothing level and the accuracy of estimated edge location. The tradeoff is also sensitive to category of edge (sharp or smooth) and SNR of the signal. Canny (1986) in his ED exploited such a tradeoff and derived an improved edge detector for noisy environment and used different size filtered derivative Gaussian kernels for gradient estimation followed by non-maxima suppression and hysteresis threshold. Though Canny ED fulfilled the localization criteria, it is degraded when the smoothing parameter $\boldsymbol{\sigma}>1$. Edge is a part of an image that contains significant dissimilarity and usually characterized by a set of parameters that can exist at any possible position and orientation.

Ideally, edge filters have to obtain the filter responses at any arbitrary position and orientation. Steerable filter is a class of filters that tuned to detect features at any possible orientation and helps in ED (Freeman and Adelson, 1991; Simoncelli and Farid, 1996). The arbitrary orientations are synthesized as a linear combination of a set of basis filters. A general approach for the design of 2-D feature detectors from a class of steerable function based on the optimization of Canny like criteria is

Corresponding Author: S. Anand, Department of Electronics and Communication Engineering, Mepco Schlenk Engineering College, 626005 Sivakasi, Tamil Nadu, India 
proposed (Jacob and Unser, 2004). Steerable filter can also be used as directional filters in the 2nd step of the contourlet implementation (Do and Vetterli, 2005; Da Cunha et al., 2006; Lu and Do, 2007) to gather local geometrical features and their orientations. Gabor wavelets are commonly used to extract the local features and simplified Gabor wavelets establish enhanced performance in ED than Canny (Choi et al., 2008). This study proposes a filter defined from quantized hyperbolic tangent profile and implemented in the steerable technique and it has the advantages of multiple orientations, good localization, and less computing cost.

\section{MATERIALS AND METHODS}

ED algorithm using steerable simplified hyperbolic tangent filter: The edges exist in an image at any scale and orientation. Thus, it is necessary to obtain the response of an edge filter at any arbitrary position and orientation. Steerable filters are such filters that are tuned to get possible orientation. One method to find the response at many orientations is to apply many versions of the same filter each different from the others by a small rotation in angle. A more efficient approach is to apply a few filters corresponding to a few angles and interpolate between the responses. Arbitrary orientation of steerable filter can be synthesized as a linear combination of a set of basis filters. The simplest illustration of the steerable principle is the directional derivative of a 2-dimensional Gaussian, $\mathrm{G}(\mathrm{x}, \mathrm{y})$ with mean $\mu$ and variance $\sigma$. The directional derivatives of the Gaussian (in polar coordinates) in $0^{\circ}$ and $90^{\circ}$ orientations are:

$$
\begin{aligned}
& \mathrm{G}_{1}^{0}(\mathrm{r}, \theta)=\cos (\theta)\left(-\mathrm{re}^{-0.5(\mathrm{r}-\mu)^{2} / \sigma^{2}}\right) \\
& \mathrm{G}_{1}^{\pi / 2}(\mathrm{r}, \theta)=\sin (\theta)\left(-\mathrm{re}^{-0.5(\mathrm{r}-\mu)^{2} / \sigma^{2}}\right)
\end{aligned}
$$

Where the subscript indicates the order of the derivative and the parenthesized superscript indicates the angle of the derivative direction. The $\sigma$ is the smoothness (or) shape parameter of the Gaussian function. It is straight forward to show that the function $G_{1}$ can be synthesized at an arbitrary orientation $\phi$ using the equation:

$$
\mathrm{G}_{1}^{\phi}(\mathrm{r}, \theta)=\cos (\phi) \mathrm{G}_{1}^{0}(\mathrm{r}, \theta)+\sin (\phi) \mathrm{G}_{1}^{\pi / 2}(\mathrm{r}, \theta)
$$

The directional derivative $G_{1}$ can be generated at an arbitrary orientation as a linear combination of the basis filters $\mathrm{G}_{1}{ }^{0}$ and $\mathrm{G}_{1}{ }^{\pi / 2}$ where the coefficients $\cos (\phi)$ and $\sin (\phi)$ are referred to as the interpolation functions.
Canny ED uses first derivative Gaussian filter to determine the gradients. However, it requires smoothing as a pre processing step to reduce noise and this blurring makes weak edges harder to detect. In practical, image edges have blurred profiles arising from the digital image acquisition process and the spatial profile of the edge filter is another factor that contributes towards noise sensitivity. SDHT has a distinguished spatial profile over Gaussian in the edge detection and satisfies the criteria like detection, localization and suppression of false edge responses. Figure 1 shows the normalized spatial profiles of Gaussian $\mathrm{g}(\mathrm{x})=\exp \left(-\mathrm{x}^{2} / 2 \sigma^{2}\right)$ with zero mean, differential of Gaussian $g^{\prime}(x)=-(x / \sigma) \exp \left(-x^{2} / 2 \sigma^{2}\right)$, differential of HBT $h^{\prime}(\mathrm{x})=\sigma-\sigma \tanh (\sigma \mathrm{x})^{2}$ and second order differential of HBT, $h^{\prime \prime}(\mathrm{x})=-(2 \tanh (\mathrm{x} \sigma)(1-\tanh$ $(\mathrm{x} \sigma)^{2} \sigma^{2}$ and it is clearly seen that the SDHT filter has narrow profile and indicate less smoothing effect for the same variance $\sigma$.

Gabor wavelets used for extracting local features in edge detection applications are common. However, extracting the Gabor features is computationally intensive Simplified version of Gabor Wavelets (SGW)

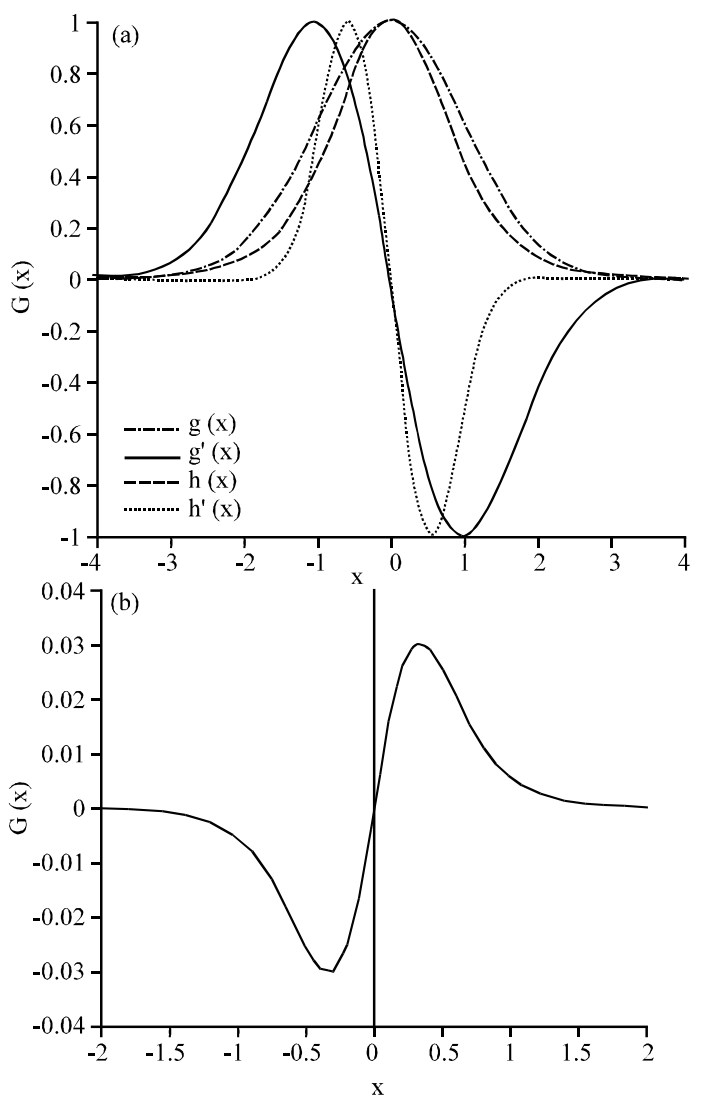

Fig. 1: a) Normalized 1D profiles of various functions; b) $1 \mathrm{D}$ profile of second order differential hyperbolic tangent filter and its five level quantization 
investigated by Choi et al. (2008) and Jiang et al. (2009) are efficient algorithms for extracting the features for edge detection. Experimental results showed that their algorithm achieved a similar performance to that using GWs while the runtime required for feature extraction using SGWs is shorter than that with GWs. Related to the SGW used for $\mathrm{ED}$, this proposed algorithm uses simplified SDHT. Since, $h^{\prime \prime}(x)$ and the Gabor functions have similar shape of 1D profile as in Fig. 1, this study proposes to use the same.

The quantization levels and values are obtained by making one of the quantization levels of SDHT is set to zero. As SDHT function is anti-symmetrical, the number of quantization levels for positive and negative values are equal. Suppose $n_{1}$ denotes the number of uniform quantization levels and the number of quantization levels becomes $2 n_{1}+1$. The quantization levels for corresponding largest magnitude $\mathrm{A}_{\max }$ :

$$
\mathrm{q}_{\mathrm{p}}(\mathrm{k})=\frac{\mathrm{A}_{\max }}{2 \mathrm{n}_{1}+1} 2 \mathrm{k}, \quad \mathrm{q}_{\mathrm{n}}(\mathrm{k})=\frac{-\mathrm{A}_{\max }}{2 \mathrm{n}_{1}+1} 2 \mathrm{k}
$$

Where $\mathrm{k}=1,2, \ldots, \mathrm{n}_{1}$ and $\mathrm{q}_{\mathrm{p}}(\mathrm{k}), \mathrm{q}_{\mathrm{n}}(\mathrm{k})$ are positive and negative quantization levels. For five quantization levels ( $\mathrm{q} 1, \mathrm{q} 2, \mathrm{q0},-\mathrm{q} 2,-\mathrm{q1})$, the simplified SDHT filter coefficients are $0.015,0.03,0,0.03,0.015$. The proposed steerable operated SDHT have following advantages due to their properties:

- Multiple orientations by steerable

- Better tradeoff between feature detection and level of smoothing by SDHT

- Low running time by simplified SDHT

\section{RESULTS AND DISCUSSION}

The proposed ED algorithm experimentally tested on images taken from University of South California Signal and Image Processing Institute (USC-SIPI) image database. Figure 2 shows the Gaussian based steerable filter and proposed SDHT filter applied on various images, i.e., boat, tree, airport and airplane $(\mathrm{F}-16)$ for the variance $\sigma=2$. While Gaussian function's, i.e., smoothness increases, edge feature's localization losses, conversely the noise influence decreases. The smoothing provides better noise control but the edge width increases and hence poor localization of edge features are visible as shown in Fig. 2. In order to compute the oriented responses of an image, the SDHT filter coefficients are convolved with any angle. The various oriented filter responses of a synthetic image size of $256 \times 256$ for various orientations, i.e., $0,45,90$ and $135^{\circ}$ are shown in Fig. 3. The edge maps of the images are obtained by applying the non-maxima suppression followed by hysteresis threshold as performed in canny edge detection. The lower and upper threshold are computed automatically for all test images. Figure 4 shows the effect of various smoothing parameter in ED. The results of Canny, steerable Gaussian and the proposed method ED for smoothing parameter $\sigma=1.5$ and 2 are shown in Fig. 4 and it is observed that increasing $\sigma$ leads to poor edge localization.

Since, the proposed method achieves better localization and smoothing due its hyperbolic tangent, it provides more visible edge features than the Canny and steerable Gaussian. Figure 5 shows the performance of the proposed $\mathrm{ED}$ on noisy image. The $\mathrm{ED}$ is operated on house image which is corrupted by Gaussian noise with $\sigma_{\mathrm{n}}=0.01$. As expected, the proposed method has many edge structures that are not found in Canny due to additional smoothness. In particular proposed operator efficiently detects the more edge features in lower roof end edges also, it has well localized edges at the cost of tiny noise features. The advantaged features are encircled in Fig. 5.

Performance comparison: To evaluate the effectiveness of the proposed method, three criterions: feature detection, noise tolerance and computational cost are considered. The parameters are:

- Mean Square Error (MSE) from reconstruction estimation measure (Agaian et al., 2010; Pellegrino et al., 2004; Nguyen and Ziou, 2000)

- PSNR from corresponding noise performances of high pass filter responses (Russo and Lazzari, 2005)

- Run time (m sec)

The reconstruction estimation image is generated by and operation of dilated edge map and original image as in Fig. 6. The MSE between original and reconstructed image provides the reconstruction estimation measure defined by; $\Sigma_{\mathrm{I}}\left(\mathrm{g}_{\mathrm{i}}-\mathrm{r}_{\mathrm{i}}\right)^{2} / \mathrm{N}$ where $\mathrm{g}_{\mathrm{i}}$ and $\mathrm{r}_{\mathrm{i}}$ are original and reconstructed estimated images, respectively. In order to recover the original image $\mathrm{g}(\mathrm{x}, \mathrm{y})$ from the edge map, the reconstruction process begins by dilating the output edge map. The original gray value at the pixel locations where dilated edge map having edge pixels $g(x, y)$ are used as interpolated value to reconstruct the image $r(x, y)$. The lower MSE of SDHT points out that the proposed algorithm has improved performance in edge detection. 

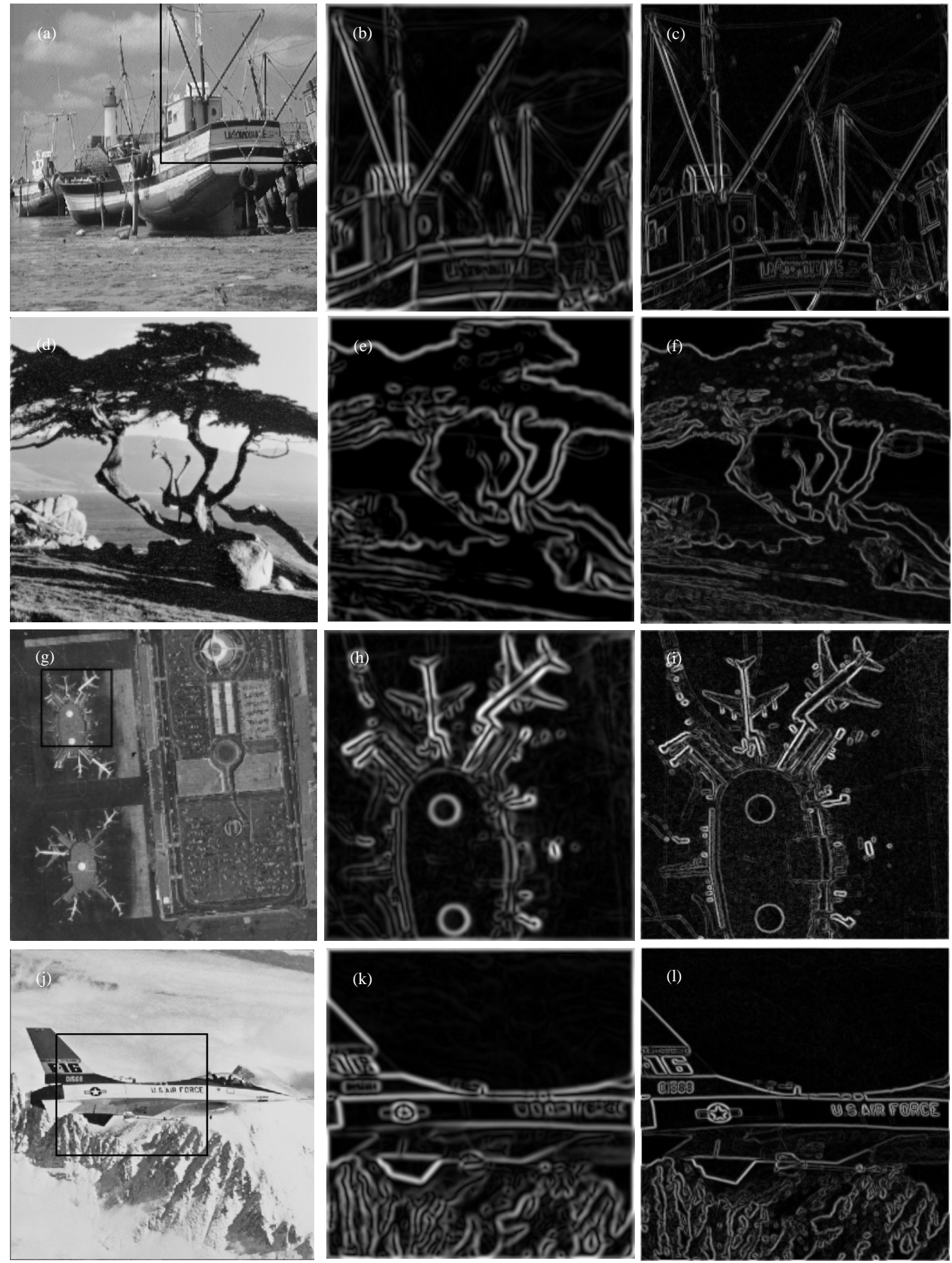

Fig. 2: The localization effect of Gaussian and hyperbolic filtering; a, d, g, j) Original images with cropped area (shown as square box); $b, e, h, k$ ) Steerable Gaussian filter responses for variance $\sigma=2 ; \mathrm{c}, \mathrm{f}, \mathrm{i}, 1)$ Steerable hyberbolic filter responses for variance $\sigma=2$

The performance comparisons of Sobel and Canny $\mathrm{ED}$ by reconstruction estimation measure for 44 different images taken from USC-SIPI shown in Fig. 7. The experimental results for $\sigma=1.5$ also proved that the hyperbolic based smoothing is better than Gaussian smoothing in particular when $\sigma>1$. Figure 8 shows the performances between the Gaussian and proposed smoothing for various $\sigma$ value, i.e., $\sigma=1,1.5,2,2.5$ and 3 . 
The noise performances are measured by adopting the method used in Russo and Lazzari (2005) where the filter responses of the noise-corrupted images compare the filter responses of the original image. The
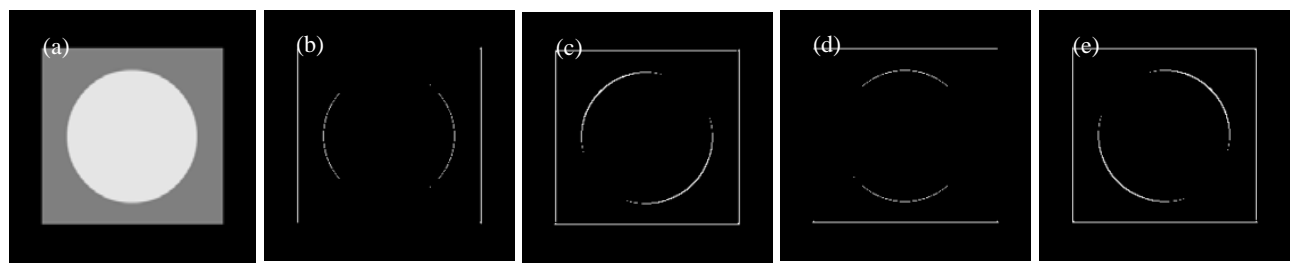

Fig. 3: Steerable filter responses; a) Original; b) Orientation $0^{\circ}$; c) Orientation $45^{\circ}$; d) Orientation $90^{\circ}$; e) Orientation $135^{\circ}$
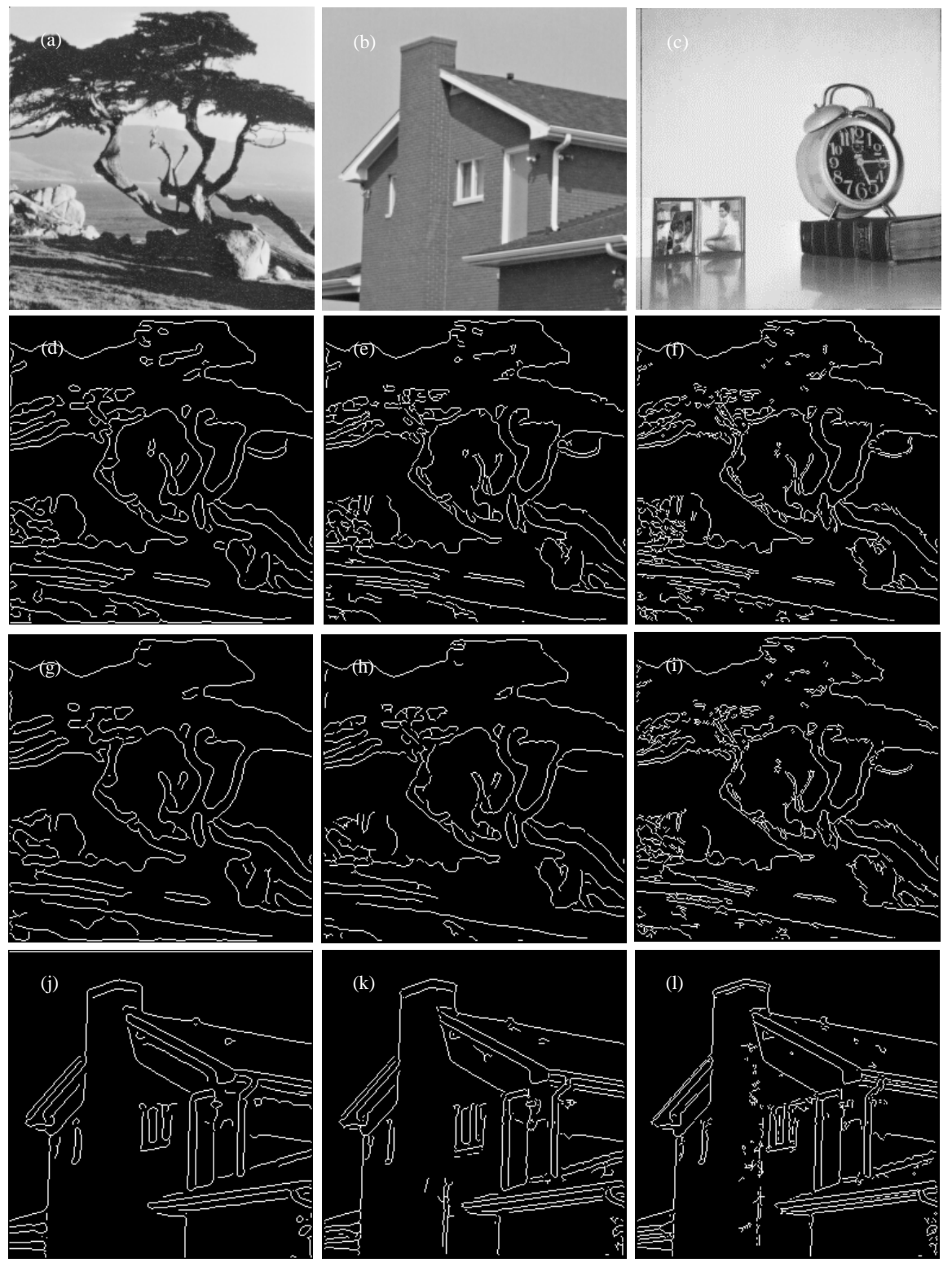

Fig. 4: Continue 

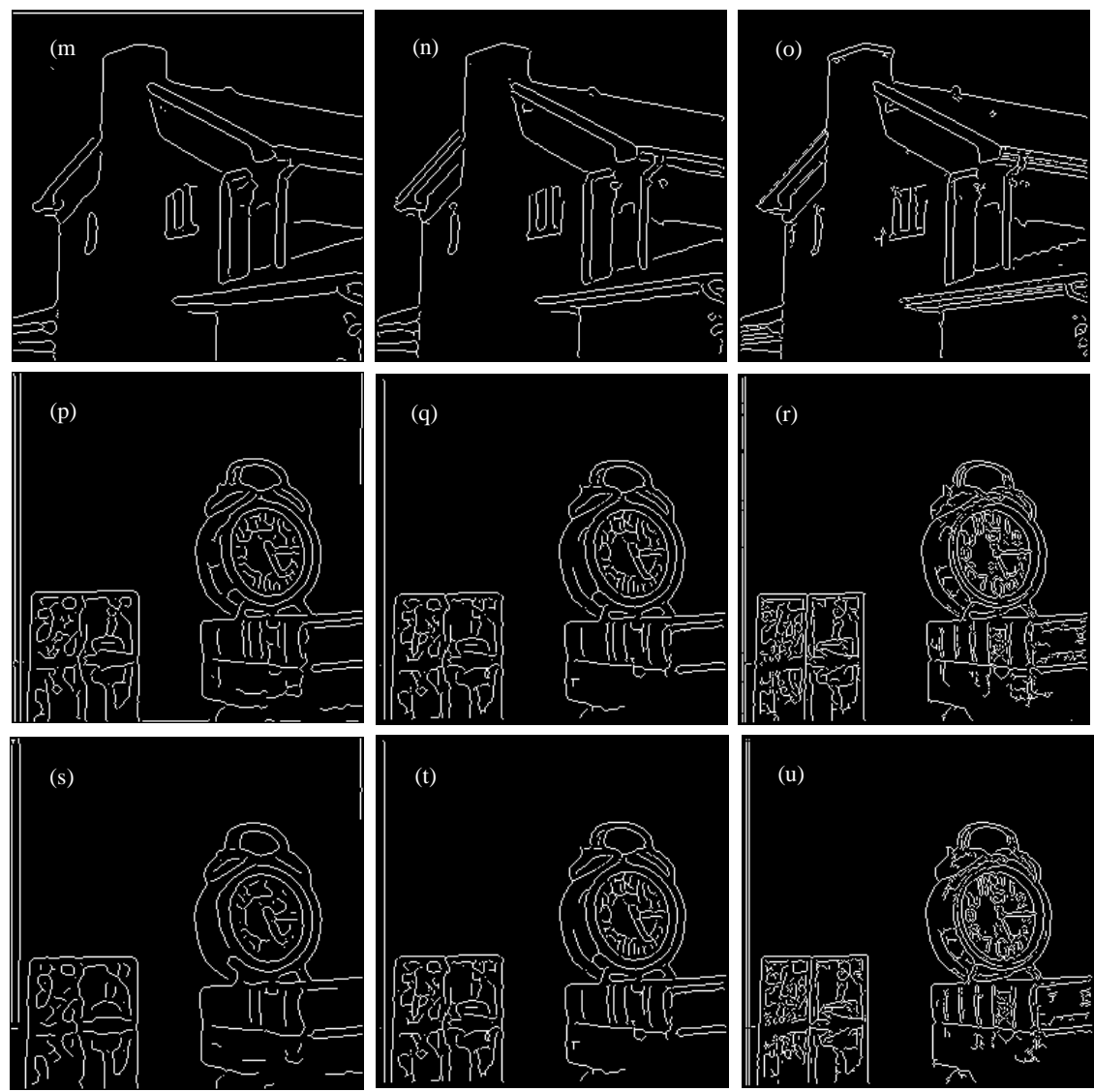

Fig. 4: Edge detection in various smoothing parameter; a-c) Original images; d, j, p) Canny with $\sigma=1.5 ; \mathrm{g}, \mathrm{m}, \mathrm{s}$ ) Canny with $\sigma=2$; e, k, q) Steerable Gaussian with $\sigma=1.5$; h, n, t) Steerable Gaussian with $\sigma=2$; f, 1, r) Proposed with $\sigma=1.5$ and $\mathrm{i}, \mathrm{o}, \mathrm{u})$ Proposed with $\sigma=2$
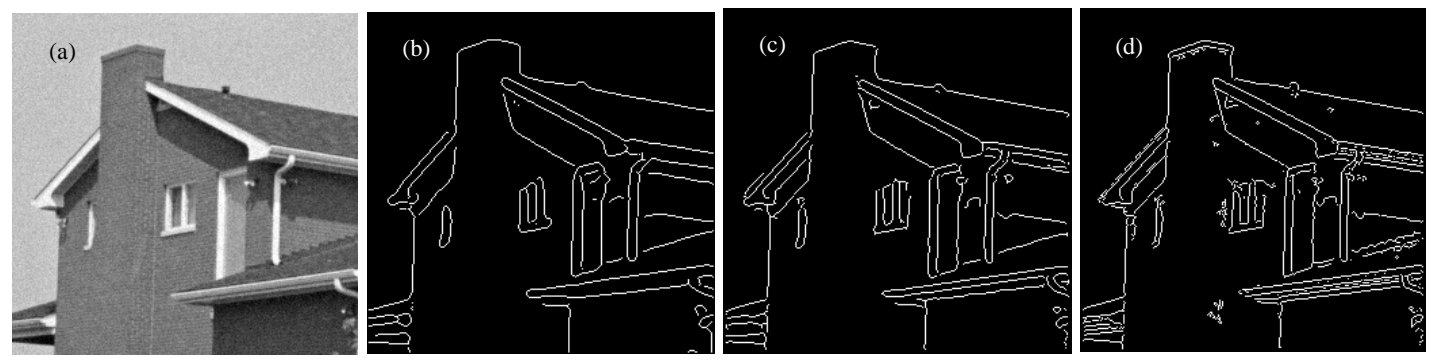

Fig. 5: Edge detection in noise environment with smoothing parameter; a) Image corrupted with noise; b) Canny; c) Steerable Gaussian; d) Proposed

corresponding noise performance is measured by means of Peak Signal to Noise Ratio (PSNR) which is defined as:

Where:

$$
\text { PSNR }=10 \log _{10} \frac{\sum_{\mathrm{i}} \Sigma_{\mathrm{j}}(\mathrm{L}-1)^{2}}{\sum_{\mathrm{i}} \Sigma_{\mathrm{j}}(\mathrm{y}(\mathrm{i}, \mathrm{j})-\mathrm{z}(\mathrm{i}, \mathrm{j}))^{2}}
$$

$\mathrm{L}=$ The number of luminance levels in an image, i.e., taken as 256 in this experiment $y(i, j)=$ The pixel luminance at location $(I, j)$ in the high frequency map of the noisy image

$z(i, j)=$ Denotes the corresponding pixel luminance in the high frequency map of the original picture

The PSNR values of restoration process of simplified Gabor Wavelet and SDHT for various images are shown in Fig. 9. 


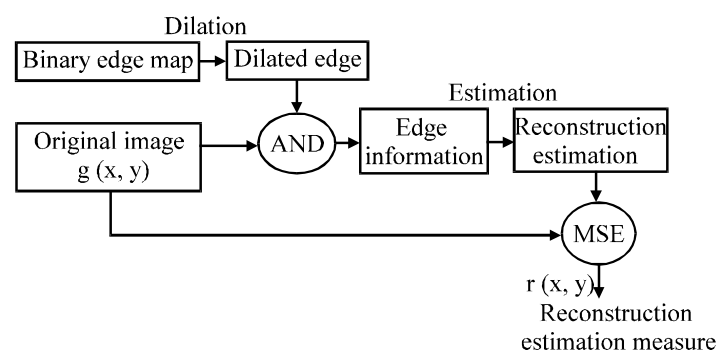

Fig. 6: Reconstruction estimation measure

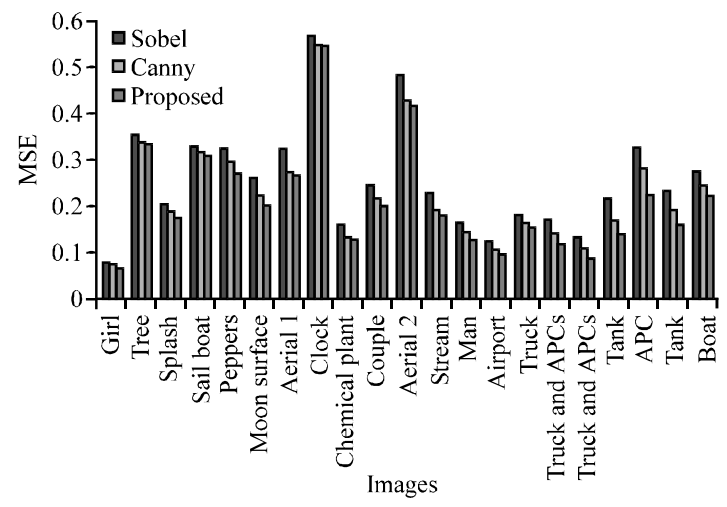

Fig. 7: Performance comparison of Sobel, Canny and Proposed method in detection

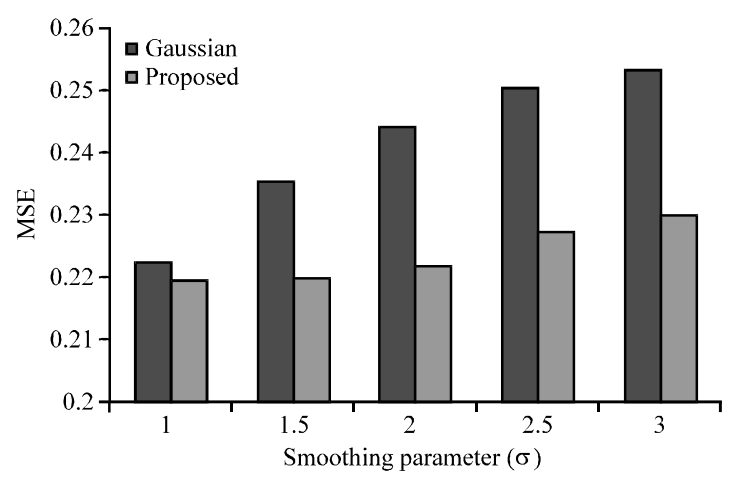

Fig. 8: Comparison of Gaussian smoothing and Proposed smoothing for various

The performance improvement given by the proposed method in terms of restoring high frequency maps from noisy conditions is very relevant and satisfactory compromise in noise sensitivity is achieved. Since, this algorithm makes use of the simplified version of hyperbolic tangent function, the computation time also get reduced. That is computing time reduced from 0.148734-0.12613 sec while running the steerable Gaussian ED and the proposed filter algorithm by Pentium 4 processor $2.4 \mathrm{GHz}$.

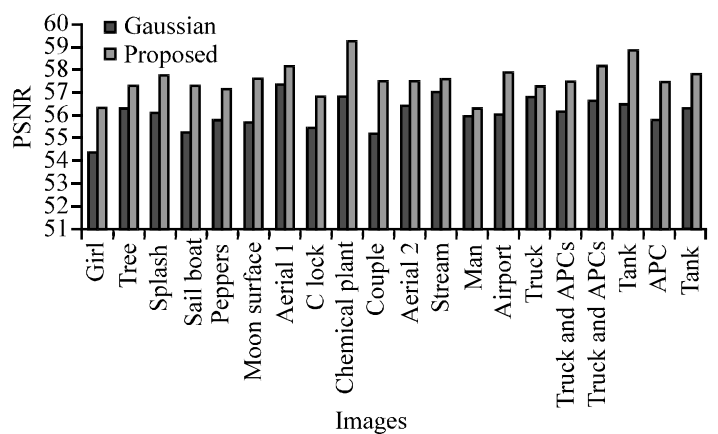

Fig. 9: Noise performance comparison of Gaussian filter and proposed SDHT filter

\section{CONCLUSION}

Simplified Second order Hyperbolic Tangent (SDHT) filter based Edge Detection (ED) is presented. The better trade-off between the level of smoothing and edge features of this algorithm provided the effective edges than common Gaussian based ED. This new algorithm captured multiple edge orientations due to steerable implementation and has less computational cost by SDHT. This study uses MSE and PSNR as quantitative measure to compare the detection and noise performances. The average is calculated by considering 44 different images. The proposed algorithm has lower MSE (0.299975) than Sobel (0.331 52) and Canny (0.30408). The PSNR have improved in proposed SDHT (57.5) than Gaussian (56.0) in noisy images. In addition, the proposed method also performs well in higher smoothing levels with less computational cost.

\section{REFERENCES}

Agaian, S.S., K.A. Panetta, S.C. Nercessian and E.E. Danahy, 2010. Boolean derivatives with application to edge detection for imaging systems. IEEE Trans. Syst. Man Cybern. Part B: Cybern., 40: 371-382.

Canny, J., 1986. A computational approach to edge detection. IEEE Trans. Patt. Anal. Mach. Intel., PAMI-8: 679-698.

Choi, W.P., S.H. Tse, K.W. Wong and K.M. Lam, 2008. Simplified Gabor wavelets for human face recognition. Pattern Recognit., 41: 1186-1199.

Da Cunha, A.L. J. Zhou and M.N. Do, 2006. The nonsubsampled contourlet transform: Theory, design and applications. IEEE Trans. Image Process., 15: 3089-3101.

Do, M.N. and M. Vetterli, 2005. The contourlet transform: An efficient directional multiresolution image representation. EEE Trans. Image Process., 14: 2091-2106. 
Freeman, W.T. and E.H. Adelson, 1991. The design and use of steerable filters. IEEE Trans. Pattern Anal. Machine Intellig., 13: 891-906.

Jacob, M. and M. Unser, 2004. Design of steerable filters for feature detection using canny-like criteria. IEEE Trans. Pattern Anal. Mach. Intell., 26: 1007-1019.

Jiang, W., K.M. Lam and T.Z. Shen, 2009. Efficient edge detection using simplified gabor wavelets. IEEE Trans. Syst. Man Cybern. Part B: Cybern., 39: 1036-1046.

Lu, Y.M. and M.N. Do, 2007. Multidimensional directional filter banks and surfacelets. IEEE Trans. Image Process, 16: 918-931.
Marr, D. and E. Hildreth, 1980. Theory of edge detection. Proc. R. Soc. Lond., 207: 187-217.

Nguyen, T.B. and D. Ziou, 2000. Contextual and noncontextual performance evaluation of edge detectors. Pattern Recognit. Lett., 21: 805-816.

Pellegrino, F.A., W. Vanzella and V. Torre, 2004. Edge detection revisited. IEEE Trans. Syst. Man Cybern. Part B: Cybern., 34: 1500-1518.

Russo, F. and A. Lazzari, 2005. Color edge detection in presence of gaussian noise using nonlinear prefiltering. IEEE Trans. Instrum. Meas., 54: 352-358.

Simoncelli, E.P. and H. Farid, 1996. Steerable wedge filters for local orientation analysis. IEEE Trans. Inform. Theory, 5: 1377-1382. 